\title{
Recent Advances in Physical Modeling \& Field Monitoring of Civil Infrastructure Systems
}

\section{Prof. Tarek Abdoun}

Natural and man-made hazards are often associated with costly damages to civil infrastructure systems, such as buildings, bridges, Levees, dams, pipelines and offshore structures of all types. The lack of high-quality field and lab data of soil system response have eluded researchers and practitioners until recently. Recent advancements in physical modeling facilities (centrifuge \& full scale) and advancement in remote sensing technology are leading to a new reality for the health assessment of soil-structure systems. This new reality is leading to a paradigm shift in the evaluation and modeling of soil-structure systems. Physical modeling, remote sensing and computational simulations are destined to replace the current empirical approaches and will ultimately become the main tool for analysis and design of soil-structure systems. The presentation will discuss the results of recent research studies utilizing physical modeling to evaluate the response of critical soil-structure systems to natural and man-made hazards. 